\title{
Modal $S$-Matrix Design of Metal Finned Waveguide Components and its Application to Transformers and Filters
}

\author{
Jens Bornemann, Senior Member, IEEE, and Fritz Arndt, Senior Member, IEEE
}

\begin{abstract}
Optimized all-metal E-plane finned waveguide components are designed with the rigorous method of modal field expansion into the ridged eigenmodes, which includes both the higher order mode interaction between the step discontinuities and the finite thickness of the fins. The design which combines the advantage of constant fin thickness with that of the optimum matching potential of different waveguide inner cross-section dimensions and fin heights achieves very broadband transformers and evanescent-mode filters with improved performance. Computer optimized data demonstrate the efficiency of the method by typical design examples: Compact transformers for WR112, WR42, WR15, and WR12 input waveguides to $E$-plane finned waveguides achieving return losses of more than $33 \mathrm{~dB}$, or $\overline{20} \mathrm{~dB}$, respectively, for the whole waveguide bands; corrugated lowpass filters designed for a cutoff frequency in the waveguide $K u^{-}(12-18 \mathrm{GHz})$ and $U$-band (40-60 GHz) with return losses of better than $20 \mathrm{~dB}$ together with a stopband attenuation of more than $60 \mathrm{~dB}$; and an evanescent-mode bandpass filter with unequal fin heights achieving $33 \mathrm{~dB}$ return loss for about $2 \%$ bandwidth at 56.5 GHz. The theory is verified by available measurements.
\end{abstract}

\section{INTRODUCTION}

$\mathbf{R}$ IDGED and all-metal finned waveguide circuits are of increasing importance for many microwave and millimeter wave devices, [1]-[19], [28], [29]. The advantges of these circuits include large single-mode broadband operation, low characteristic impedances, and, in its finned version with thin ridges, the possibility of low-cost, low-loss E-plane integrated circuit designs. Of particular importance are the transitions between ridged or finned waveguides of different gap height and waveguide inner cross-sections as well as the junction to the rectangular input waveguide [1], [2], [6]-[9], [12]. Moreover, the finned waveguide circuit may advantageously be utilized for compact and easy-to-fabricate evanescent-mode filter designs [10], [11], [14]-[18], [28], [29].

Based on Cohn's transmission-line approximation [20], Hensperger [2] presented a transformer from a WR-112 rectangular to the Airtron ARA-133 double-ridged wave-

Manuscript received August 19, 1991; revised January 25, 1992.

J. Bornemann is with the Department of E.C.E., University of Victoria, P.O. Box 3055, Victoria, BC V8W3P6, Canada.

F. Arndt is with the Microwave Department, University of Bremen, Fachbereich 1, Kufsteiner Str. NWI, P.O. Box 330440, W-2800, Germany.

IEEE Log Number 9108322 guide [19]. That design includes a continuous transition from the input waveguide to the smaller ridged waveguide cross-section. A constant ratio (of 0.25 ) in ridge thickness to waveguide width has been utilized which requires also the ridge thickness to be tapered along the whole length of the transition. Therefore, the design is considered to be not very appropriate for the modern fabrication methods for microwave and millimeter-wave E-plane integrated circuits, such as etching or computer controlled milling techniques. A stepped transformer with constant ridge thickness has been presented in [12], but the transformer housing dimensions are kept unchanged. Hence, the advantage of the high bandwidth potential inherent in a proper choice of the waveguide cross-sections is not utilized.

Evanescent-mode filters [10], [11], [15]-[18], [28] are of particular importance due to several advantages over the conventional filter types e.g. compactness and wide stopbands. In [10], [16], bilateral or unilateral ridge waveguide sections are used to construct distributed shunt capacitances in lowpass filter designs. More recently, nontouching unilateral E-plane fins of negligible thickness with and without a dielectric layer [11] have been applied for the design of evanescent-mode bandpass filters. As dielectric layers cause additional losses, all-metal circuit designs are often preferred [11], [14]-[18]. On the other hand, the usual thick ridge waveguide, capacitive screw, and round post filter elements [14]-[17], [28] are difficult to fabricate at low cost and to mass-produce. An all-metal finned waveguide circuit design [13], [18], may help to avoid this disadvantage. The full design potential inherent to a suitable choice of the evanescent-mode waveguide cross-section, however, is not yet utilized in [13], [18] and the low-pass filter design of [28] still uses equal slot widths in the filter section. The inclusion of the additional optimization parameters, i.e. different cross section dimension and different slot widths, achieves the design of finned waveguide components of improved performance.

This paper describes a rigorous field theory design of broadband and compact all-metal finned waveguide components of the generalized structure of Fig. 1. The method may be applied for the design of transformers with optimum stepped transitions for both the fins and the different outer cross-sections from the input waveguide to the 


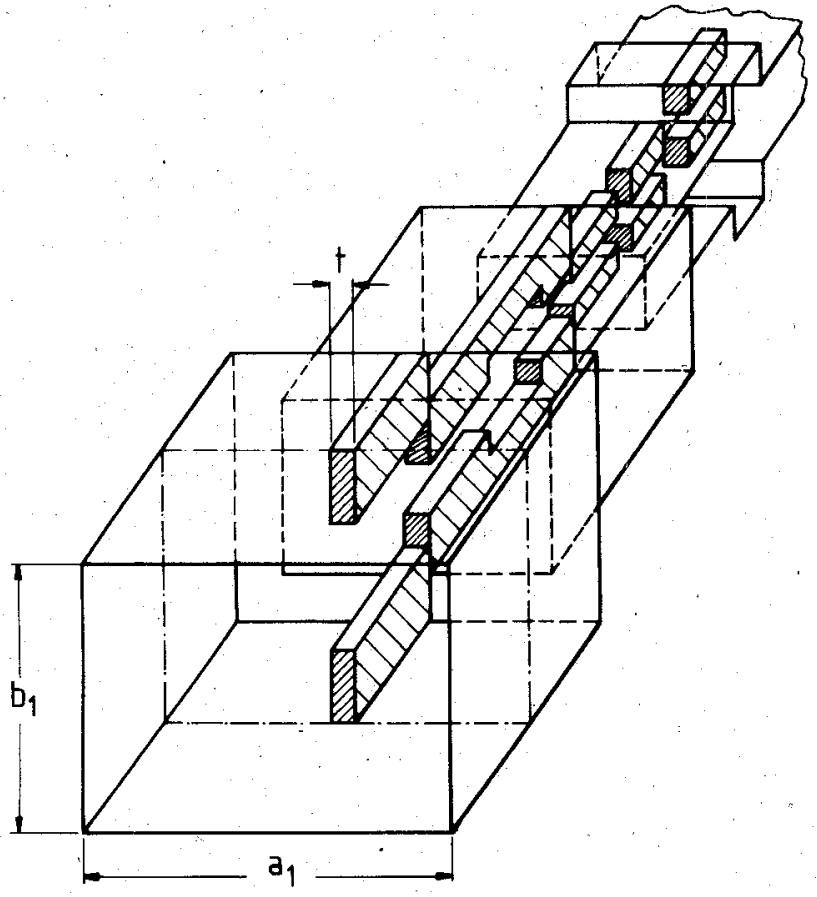

Fig. 1. General E-plane all-metal finned waveguide structure under investigation with different waveguide cross-sections for components of improved performance (e.g., transformers for increased frequency bands and evanescent-mode filters with increased rejection quality).

smaller housing of the ridged waveguide. This design combines the advantage of stepped fins of constant thickness, well suited to the printed E-plane technology; with that of the additional matching potential of the different cross-section dimensions, which achieves an improved return loss behavior and a compact structure. Furthermore, the method is applied to provide the efficient computer-aided design of improved types of evanescent-mode finned waveguide filters.

Based on bilateral all-metal E-plane fins, the design combines the well-known properties of the waveguide E-plane integrated-circuit technology [6], [9], with the advantages of the evanescent-mode concept, [10], [11], [14]-[18], and the low-insertion loss quality resulting from the complete absence of supporting dielectrics, [12], [26]. The proposed structure, where the finite fin thickness is included in the design, achieves relatively large slot widths of about one quarter of the waveguide height in the below cutoff section and leads to filter characteristics with high skirt selectivity and wide stopbands. Additional finned waveguide transformer sections are utilized for an optimum match of the input/output waveguide to the corrugated ridged structure within an appropriately reduced cross-section waveguide which achieves an increased stopband attenuation level.

Various approaches, such as the mode-matching, spectral-domain and the variational analysis, have been reported for the characterization of the ridge or finned waveguide discontinuity [6], [7], [11]-[13], [28]. The effects of both the accurate field description of the ridge waveguide sections and the metallization thickness have turned out to be important for reliable designs with cascaded all-metal finline discontinuities. In this paper, the modematching technique combined with the transverse resonance field-matching technique for the cross-section eigenfunctions with propagating partial waves [29] is used which yields the advantage of a well-conditioned determinant function without poles. Moreover, this method has turned out to be particularly appropriate for all-metal finned waveguide structures with moderate fin thicknesses and slot widths which are treated in this paper.

The generalized modal scattering matrix technique is applied for calculating the cascaded discontinuities. This allows the higher-order mode coupling effects to be taken into account in the design. For computer optimization, the evolution strategy method [12], [26], i.e. a suitably modified direct search procedure, is applied where no differentiation step in the optimization process is necessary, and hence the problem of local minima may be circumvented. The exact design theory presented enables high precision manufacturing by etching or milling techniques without the necessity of post assembly 'trial and error' adjustment methods. Design examples for optimized' structures will demonstrate the efficiency of the design method. The theory is verified by available measured results.

\section{THEORY}

For the computer-aided design of the all-metal E-plane finned waveguide transformers and evanescent-mode filters (Fig. 1), the modal $S$-matrix method [12], [18], [26] is applied. The structure is decomposed into two key building blocks: rectangular waveguide double-step junction discontinuity [27], and the step discontinuity rectangular waveguide to the finite-length ridge section (Fig. 2). Combination with the modal scattering matrices of the corresponding intermediate homogeneous waveguide sections of finite or zero lengths, respectively, yields the total scattering matrix of the transformer or filter, respectively.

The electromagnetic field in the subregions $i=\mathrm{O}, \mathrm{I}, \mathrm{II}$ (Fig. 2)

$$
\begin{aligned}
\vec{E}^{i} & =\nabla \times\left(A_{H z}^{i} \vec{e}_{z}\right)+\frac{1}{j \omega \epsilon} \nabla \times \nabla \times\left(A_{E z}^{i} \vec{e}_{z}\right) \\
\vec{H}^{i} & =\nabla \times\left(A_{E z}^{i} \vec{e}_{z}\right)-\frac{1}{j \omega \mu} \nabla \times \nabla \times\left(A_{H z}^{i} \vec{e}_{z}\right)
\end{aligned}
$$

is derived from the $z$-components of two vector potentials

$$
\begin{aligned}
\vec{A}_{H z}^{i}= & \sum_{q=1}^{\infty}\left(\sqrt{Z_{H q}^{i}}\right) \cdot T_{H q}^{i}(x, y) \\
& \cdot\left[V_{H q}^{i} \exp \left(-j k_{z H q}^{i} z\right)+R_{H q}^{i} \exp \left(+j k_{z H q} z\right)\right] \\
\vec{A}_{E z}^{i}= & \sum_{p=1}^{\infty}\left(\sqrt{Y_{E p}^{i}}\right) \cdot T_{E p}^{i}(x, y) \\
& \cdot\left[V_{E p}^{i} \exp \left(-j k_{z E p}^{i} z\right)-R_{E p}^{i} \exp \left(+j k_{z E p} z\right)\right],
\end{aligned}
$$




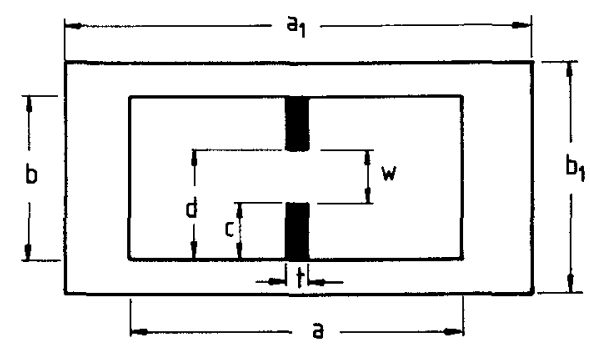

(a)

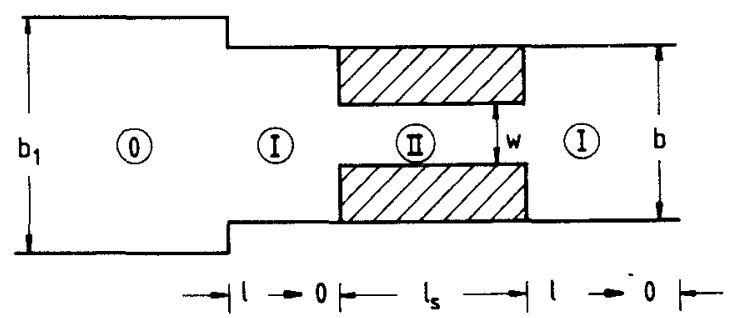

(b)

Fig. 2. Discontinuities for the field theory treatment. (a) Cross-sectional dimensions of the finned waveguide. (b) Rectangular waveguide doublestep junction discontinuity and step discontinuity rectangular waveguide to the finite-length ridge waveguide section.

with the wave impedances

$$
\begin{gathered}
Z_{H q}^{l}=\left(\omega \mu_{0}\right) /\left(k_{z H q}^{l}\right)=1 / Y_{H q}^{i}, \\
Y_{E p}^{l}=\left(\omega \epsilon_{0}\right) /\left(k_{z E p}^{i}\right)=1 / Z_{E p}^{i} .
\end{gathered}
$$

$V_{H, E}^{i}, R_{H, E}^{i}$ are the TE- and TM-mode wave amplitudes of the forward and backward waves, respectively, which have to be related to each other at the corresponding discontinuity. This will yield the corresponding scattering matrix relations. $k_{z}$ are the propagation factors, and $T_{H q}^{i}$, $T_{E p}^{i}$ are the cross-section eigenfunctions of the corresponding waveguide structures under consideration, i.e. finned waveguide [12], [18], and rectangular waveguide [12], [27]. For completeness, the expressions for the finned waveguide are given in the Appendix.

For the E-plane finned or ridged waveguide eigenvalue problem (Fig. 2, region II), the transverse resonance method is used [12], [18], [21]-[23], [29], see Appendix. This procedure reduces the size of the characteristic matrix equation to a quarter of the original size. Furthermore, it makes the method very flexible because an arbitrary number of subregions (e.g., fin-lines with multiple inserts) may be easily taken into account in the matrix system, simply by multiplying the additional transmission-line matrices of the corresponding insert subregions. The main advantage of the transverse resonance method described, e.g., compared with the conventional modematching [24] or resonator techniques [25], however, is the fact, that there are no poles in the determinant function of the resulting characteristic matrix equation [29]. Therefore, the search algorithm in order to find a given number of subsequent eigenvalues (i.e., the propagation constants for a given frequency, or, like in the present case, the cutoff frequencies) can be simplified, and the risk of omitting eigenvalues is very low. This compensates for the disadvantage of the method (i.e. the required identical number of expansion terms in the subregions), especially since the cross-section structures involved in the investigation of this paper need no special selection of the expansion terms.

In order to calculate the modal scattering matrix of the key-building block discontinuities of the transformer or filter components (Fig. 1), respectively, directly by the corresponding field matching relations of the wave amplitude coefficients according to (2), the cross-section eigenfunctions are suitably normalized so that the power carried by a given mode is

$$
\begin{aligned}
P_{M}^{i} & =\int_{F^{i}}\left(\vec{E}_{M}^{i} \times \vec{H}_{M}^{i *}\right) \vec{e}_{z} d F \\
& =\sqrt{Z_{M}^{i}} \sqrt{Y_{M}^{i} *} \int_{F^{i}}\left(\operatorname{grad} T_{M}^{i}\right)^{2} d F \\
& =\left\{\begin{array}{c}
1 W, \text { propagat. modes } \\
j W, \text { evanesc. TE modes } \\
-i W . \text { evanesc. TM modes. }
\end{array}\right.
\end{aligned}
$$

Matching the tangential field components of regions I and II at the common interface (Fig. 2(b)) yields the modal scattering matrix of the step discontinuity waveguide to ridged or finned waveguide.

$$
\left[\begin{array}{l}
\left(R^{I}\right) \\
\left(V^{I I}\right)
\end{array}\right]=(S)\left[\begin{array}{c}
\left(V^{I}\right) \\
\left(R^{I I}\right)
\end{array}\right]
$$

where the submatrices are already described in [12]. The modal scattering matrix of the rectangular waveguide double-step discontinuity is already given in [27]. The series of step discontinuities, for a complete transformer or filter structure, is calculated by direct combination of the single modal scattering matrices [12].

The computer-aided design is carried out by an optimization program applying the evolution strategy method [12], [18], [26]. An error function is maintained with respect to a parameter vector, which contains the slot widths and section lengths, until a specified characteristic is achieved over the desired frequency range. The number of TE-modes, TM-modes, and cross-sectional expansion terms [12] are chosen to be 5, 2, 8 for the optimization, and $12,7,8$ for the final analysis, respectively.

The numbers have been obtained by checking the convergence behavior against measurements [12], [29], cf. also Fig. 3. In Fig. 3, the dashed curve $(5,2,8)$ in comparison with the solid curve $(12,7,8)$ demonstrates the significant influence of the higher order modes on the correct prediction of the return loss peaks which may be particularly important for narrow-band designs. (For the dimensions of Figs. 3, 4, 6, 7, 8, 9, 10(a)-(b) and 11 see Table I in the Conclusion section of this paper.) 


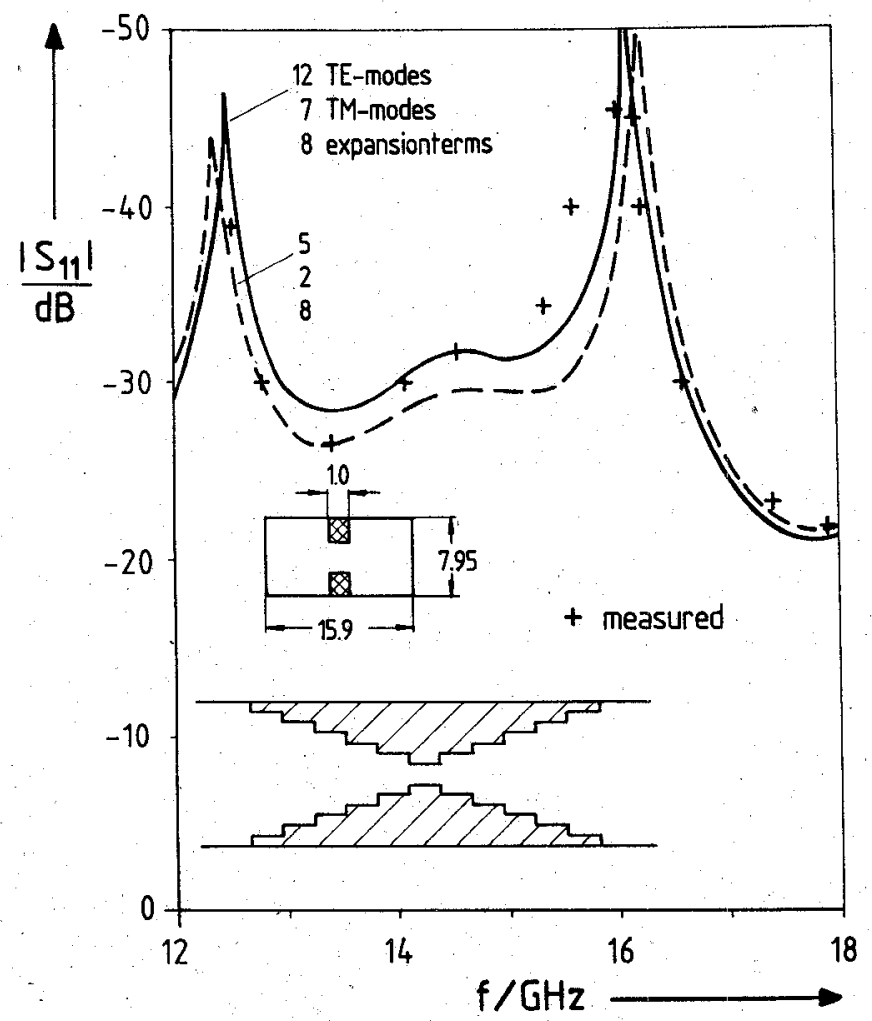

Fig. 3. Input return loss $1 /\left|S_{11}\right|$ in $\mathrm{dB}$ as a function of frequency of an optimum stepped $K u$-band $(12-18 \mathrm{GHz})$ ridged waveguide transformer with two symmetrical five-step sections and with constant waveguide inner crosssection dimensions. Comparison between measurements $(+++)[12]$ and theory. Demonstration of the convergence behavior by the characteristic (dashed curve) obtained by a reduced number of modes. Dimensions cf. Table I.

\section{RESULTS}

Fig. 4 shows the comparison between Hensperger's [2] and our design, at the example of the five-step WR-112 $(7.05-10 \mathrm{GHz})$ waveguide to ARA-133 ridged waveguide transformer [19]. Additional to the advantage of constant ridge thickness, the double-stepped transformer design achieves a reduction of the maximum VSWR within the whole frequency range; moreover, the overall transformer length is slightly decreased from about 48 to $46 \mathrm{~mm}$. The minimum return loss is better than $36 \mathrm{~dB}$ for the optimized structure which may demonstrate the advantage of the computer-aided field theory design.

Fig. 5 presents the comparison of the results of our theory with measurements of [13] and calculations of [13] and [28], for the example of a cascaded E-plane ridged waveguide discontinuity. In our theory, the structure has been calculated with 20 TE, 15 TM modes and 10 expansion terms. Good agreement with the measurements of [13] may be stated, especially in the lower frequency region. In contrast to [13] and [28], however, we calculated two additional resonance peaks. As these peaks mainly occur beyond $-40 \mathrm{~dB}$ it should be noted that the actual structure contains losses which may limit the dynamic range of the measured resonances to values below -40 dB. Moreover, the validity of our theory (including res-

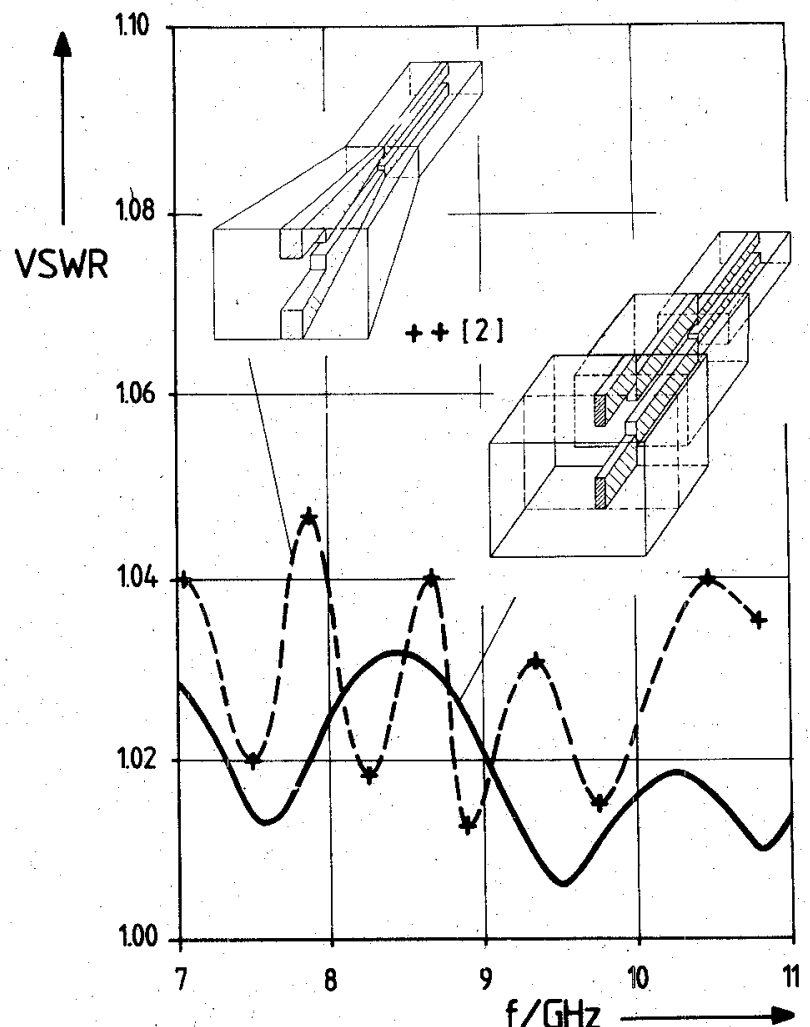

Fig. 4. Input VSWR versus frequency of an optimum five-step WR-112 (7.05-10 GHz) waveguide to ARA-133 ridged waveguide transformer (solid line); comparison with Hensperger's linearly tapered ridge thickness design (dashed line). Dimensions cf. Table $\mathbf{I}$.



Hig. 5. Transmission coefficient of a cascaded E-plane ridged waveguide discontinuity [13]: $a=22.86 \mathrm{~mm}, b=10.16 \mathrm{~mm}, s=4.114 \mathrm{~mm}, t=$ $2.057 \mathrm{~mm}, l_{r}=1.524 \mathrm{~mm}, l_{c}=12.192 \mathrm{~mm}$. Comparison with the results of [13] and [28]. 


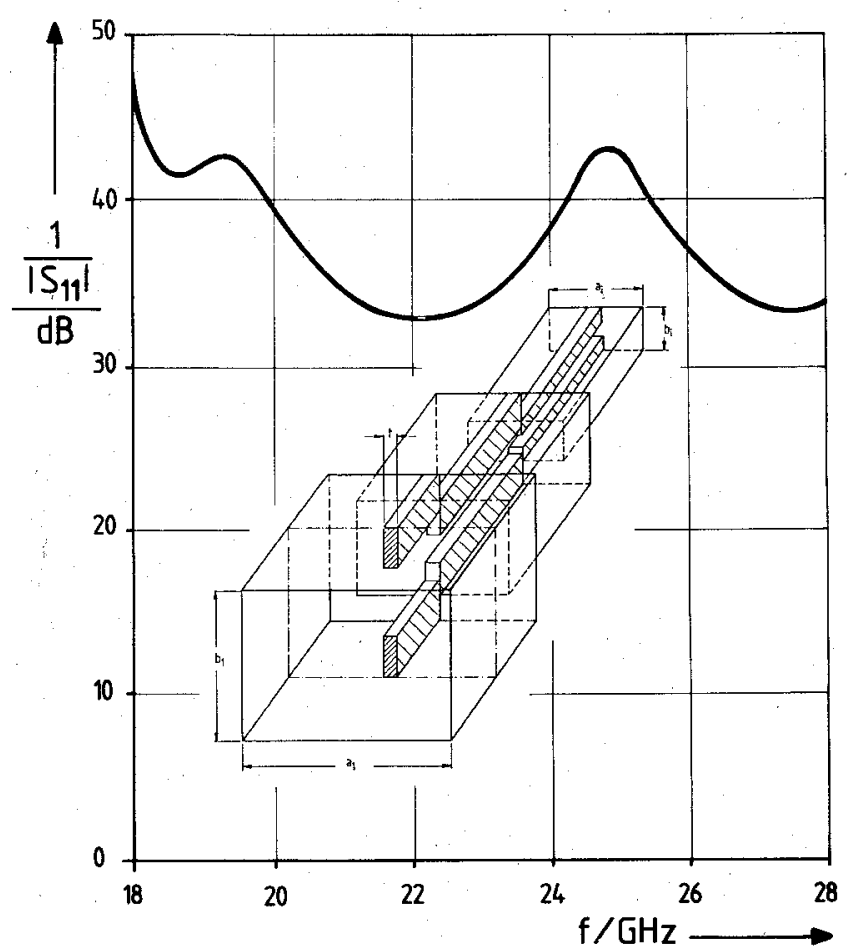

Fig. 6. Input return loss $1 /\left|S_{11}\right|$ in $\mathrm{dB}$ as a function of frequency of a stepped E-plane all-metal finned waveguide transformer of different inner cross-sections. Analysis of a frequency-scaled version of the optimized configuration of Fig. 4 for a WR-42 input waveguide ( $K$-band: $18-26 \mathrm{GHz}$ ). Dimensions cf. Table I.

onance peaks within the range of $20 \cdots 40 \mathrm{~dB}$ ) has already been demonstrated by measurements in [29].

In Fig. 6, the result of a frequency-scaled version of the optimized configuration of Fig. 4 is presented for a WR-42 input waveguide ( $K$-band: $18-26 \mathrm{GHz}$ ). The good return loss behavior is maintained beyond the limit of the waveguide band of the input waveguide. The slight reduction in return loss (minimum value $33 \mathrm{~dB}$ ), as compared with the design of Fig. 4 , is due to the fact that a modified ratio of the ridge thickness to waveguide width, $\mathrm{t} / \mathrm{a}$, has been chosen in order to obtain an adequately manufacturable ridge thickness $(t=2.4 \mathrm{~mm})$.

The return loss of an optimized seven-step transformer from WR-15 (50-75 GHz) input waveguide to a finned waveguide with the inner dimensions of a standard $\mathbf{W} R-12$ waveguide housing $(3.099 \mathrm{~mm} \times 1.549 \mathrm{~mm})$, a fin thickness of $0.1 \mathrm{~mm}$ and a slot width of $w=0.4 \mathrm{~mm}$ is shown in Fig. 7 (solid lines). The design achieves a return loss of more than $20 \mathrm{~dB}$ within the whole frequency band of an octave (50-100 GHz). For this broadband single-mode design, the next higher propagating modes do not appear up to $119.6 \mathrm{GHz}$. The sensitivity of the performance of the optimized transformer to dimensional tolerances is also demonstrated in Fig. 7: The dashed and dash-dotted curves show the return loss for a worst-case simulation of typical etching tolerances (section length and slot width variation $\pm 0.02 \mathrm{~mm}$ ). An optimized five-step transformer from the WR-12 input waveguide directly to its finned section $(t=0.1 \mathrm{~mm}, w=0.4 \mathrm{~mm})$ achieves a return loss of more than $30 \mathrm{~dB}$ within $60-97 \mathrm{GHz}$ (Fig. 8).

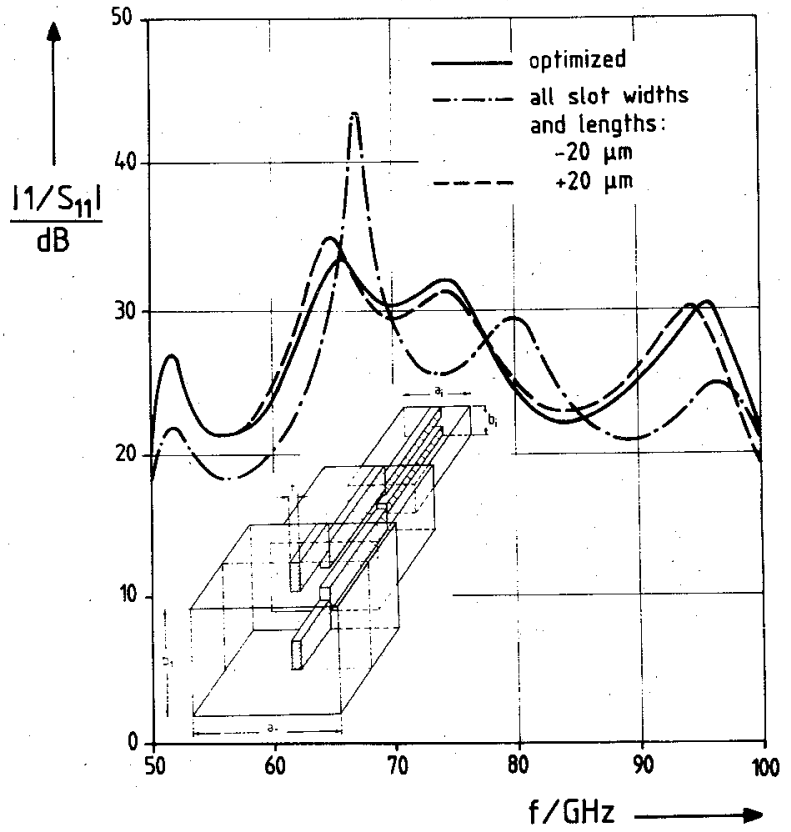

Fig. 7. Input return loss $1 /\left|S_{11}\right|$ in $\mathrm{dB}$ as a function of frequency of an optimized seven-step transformer from WR-15 (50-75 GHz) input waveguide to a finned waveguide with the inner dimensions of a standard WR12 waveguide housing $(3.099 \mathrm{~mm} \times 1.549 \mathrm{~mm})$, a fin thickness of $0.1 \mathrm{~mm}$ and a slot width of $w=0.4 \mathrm{~mm}$ (solid line). Sensitivity to dimensional tolerances (dashed and dash-dotted curves) for a worst-case simulation of section length and slot width variation $\pm 0.02 \mathrm{~mm}$. Dimensions $\mathrm{cf}$. Table I.

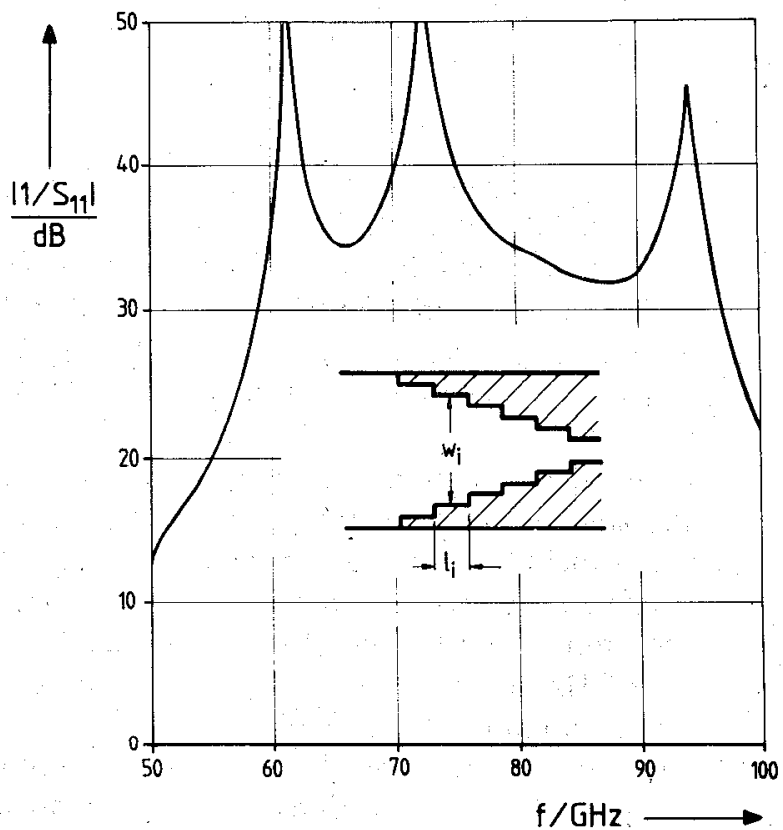

Fig. 8. Input return loss $1 /\left|S_{11}\right|$ in $\mathrm{dB}$ as a function of frequency of an optimized five-step transformer from the WR-12 input waveguide directly to its WR-12 finned section $(t=0.1 \mathrm{~mm}, w=0.4 \mathrm{~mm})$. Dimensions cf. Table I.

Ridged or finned waveguide transformer sections may be advantageously utilized for an optimum match of the desired input/output waveguide to a corrugated finned waveguide structure, and hence may achieve improved 


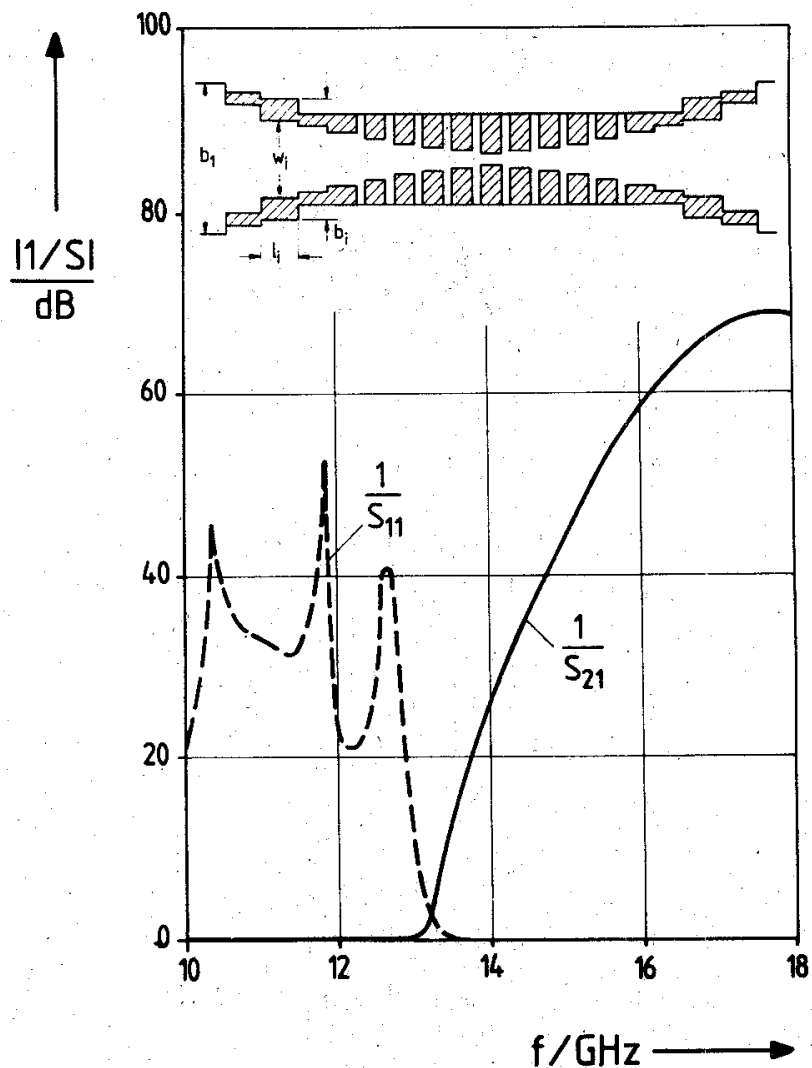

Fig. 9. Insertion loss $1 /\left|S_{21}\right|$ (solid line) and input return loss $1 /\left|S_{11}\right|$ (dashed line) in $\mathrm{dB}$ of an optimized quasi-lowpass filter with an input/ output WR-75 waveguide designed for a passband from $10 \mathrm{GHz}$ (cutoff frequency of the WR-75 waveguide band) to $13.2 \mathrm{GHz}$. Dimensions cf. Table I.

filter designs. Fig. 9 shows an optimized quasi-lowpass filter with an input/output WR-75 waveguide $(19.05 \mathrm{~mm}$ $\times 9.525 \mathrm{~mm}$ ) designed for a passband from $10 \mathrm{GHz}$ (cutoff frequency of the WR-75 waveguide band) to 13.2 $\mathrm{GHz}$, a minimum passband return loss of $20 \mathrm{~dB}$, and a desired stopband attenuation of $60 \mathrm{~dB}$ between 16.5 and $18 \mathrm{GHz}$.

The design data are transferable into other common waveguide bands by suitable frequency scaling relations which should include the metal fin thickness. Fig. 10(a) shows a related design with an input/output WR-22 waveguide $(5.69 \mathrm{~mm} \times 2.845 \mathrm{~mm})$ for a passband from 33 $\mathrm{GHz}$ to $43.9 \mathrm{GHz}$, a return loss of $20 \mathrm{~dB}$ and a maximum stopband attenuation of about $68 \mathrm{~dB}$.

The stopband characteristic of filters may be improved by additional E-plane corrugations. Fig. 10(b) presents the results for the filter example of Fig. 10(a) but with additionally optimized E-plane corrugations. The frequency band of a stopband attenuation of more than 60 $\mathrm{dB}$ is increased whereas the return loss is still more than $20 \mathrm{~dB}$.

Fig. 11 shows the results of an optimized evanescentmode bandpass filter with the passband at about $56.5 \mathrm{GHz}$. As different fin widths $w_{i}$ are included in the design procedure, an excellent filter characteristic is obtained. Although this design requires expanded computer resources

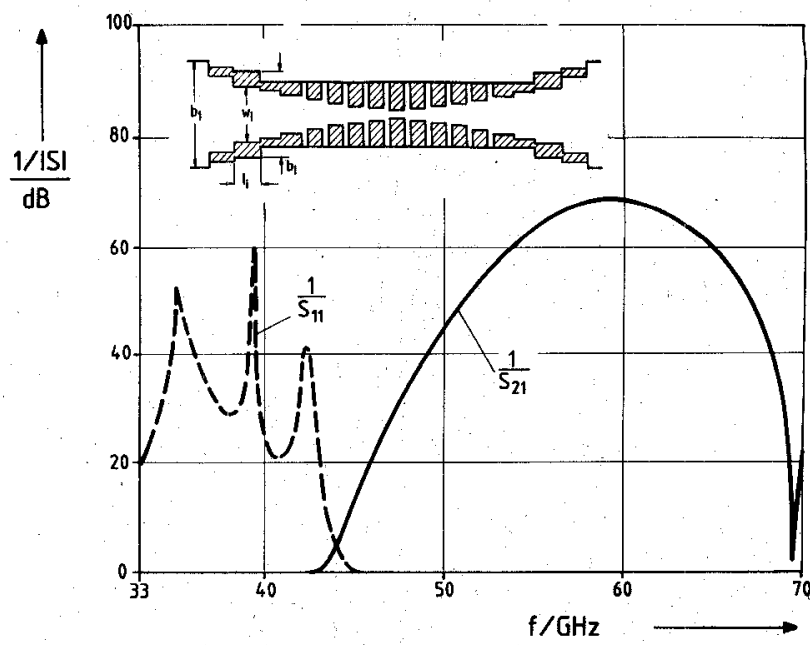

(a)

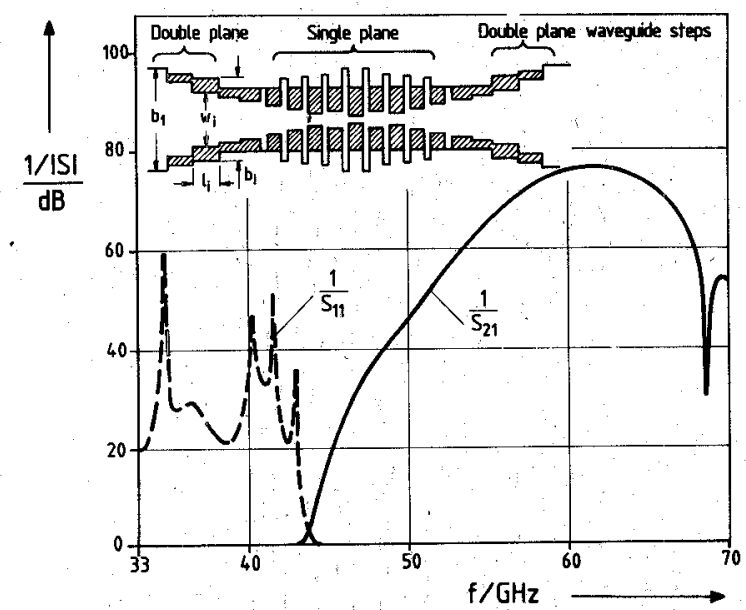

(b)

Fig. 10. Insertion loss $1 /\left|S_{21}\right|$ (solid line) and input return loss of $1 /\left|S_{11}\right|$ (dashed line) in $\mathrm{dB}$ of an optimized quasi-lowpass filter with an input/output WR-22 waveguide designed for a passband from $33 \mathrm{GHz}$ to $43.9 \mathrm{GHz}$. (a) Frequency scaled version of Fig. 9. Dimensions cf. Table I. (b) Optimized version with additional E-plane step discontinuities in the inner waveguide section. Dimensions cf. Table I.

compared to its constant-width counterpart [30], it offers more flexibility for the optimization procedure to compensate for the waveguide double-plane step discontinuity without the need for additional transformer sections as, e.g., in [28].

For theory-practice comparisons, beyond those presented in Figs. 3, 5, and especially related to the type of filter structure discussed here, the reader is referred to [29], [30], where a designed three-resonator evanescentmode finned waveguide filter has been realized which shows excellent agreement between measurements and the theoretical predictions. Moreover, the mode-matching and generalized scattering matrix technique applied in this paper has been already verified by many other filter or even more complicated diplexer examples, such as rectangular iris coupled resonator filters [31] or compact low-insertion-loss waveguide diplexers [32]. 


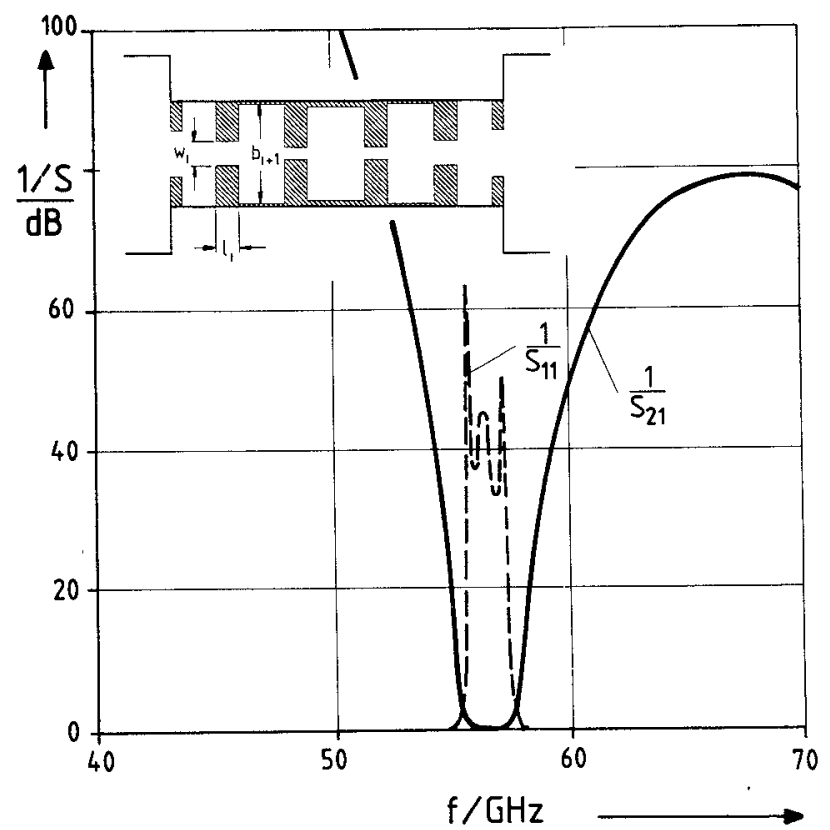

(a)

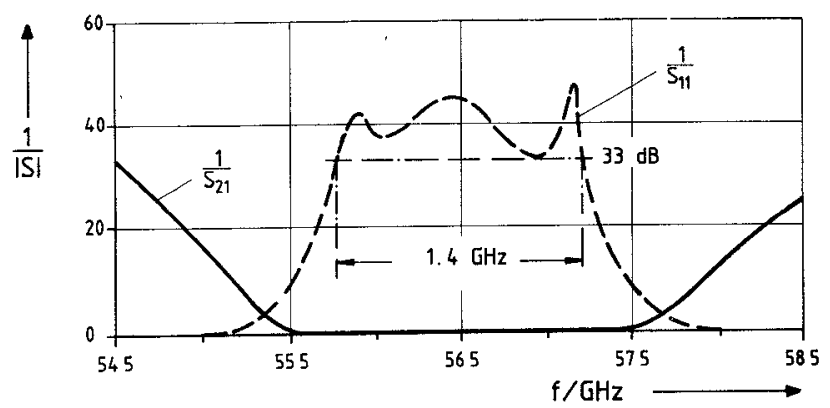

(b)

Fig. 11. Insertion loss $1 /\left|S_{21}\right|$ (solid line) and input return loss $1 /\left|S_{11}\right|$ (dashed line) in $\mathrm{dB}$ of an optimized evanescent mode filter with an input output WR-19 waveguide designed for a passband at about $56.5 \mathrm{GHz}$. Dimensions cf. Table I. (a) Frequency range $40 \cdots 70 \mathrm{GHz}$. (b) Expanded view.

\section{ConClusion}

The rigorous modal S-matrix method presented achieves the exact computer-aided design of broadband all-metal E-plane finned waveguide transformers which combines the advantage of constant ridge thickness, well suited to the printed E-plane technology, with that of the additional matching potential of different inner waveguide dimensions. Moreover, the efficient computer-aided design method may be applied for the construction of improved types of evanescent-mode finned waveguide filters. Additional finned waveguide transformer sections are uti- lized for an optimum match of the input/output waveguide to the corrugated ridged structure of reduced cross-section and the additional design potential of E-plane corrugations and unequal fin width is taken into account. This achieves increased return loss behavior within the passband and a high stopband attenuation level. The design data are transferable into other frequency bands of interest. Since the theory includes the finite thickness of the fins as well as the higher order mode interaction at all discontinuities, the available measured results verify the theory by good agreement.

TABLE I

Optimized Design Data for E-Plane Finned Waveguide Transformers and Filters

Fig. 3: Transformer, $t=1 \mathrm{~mm}$

Input waveguide: $a_{1}=15.9 \mathrm{~mm}, b_{1}=7.95 \mathrm{~mm}$

$l_{1}=5.980 \mathrm{~mm}$;

$l_{3}=7.370 \mathrm{~mm}$

$l_{5}=5.620 \mathrm{~mm}$

$$
\begin{aligned}
& w_{1}=6.670 \mathrm{~mm} ; \\
& w_{3}=4.290 \mathrm{~mm} ; \\
& w_{5}=2.440 \mathrm{~mm} ;
\end{aligned}
$$

\begin{tabular}{|c|c|c|c|c|c|c|}
\hline$a_{i} / \mathrm{mm}:$ & 28.254, & 27.761 & 27.268 & 26.775 & 26.282 & 26.035 \\
\hline$b_{1} / \mathrm{mm}:$ & 12.568 & 12.456 & 12.345 & 12.233, & 12.121, & 12.065 \\
\hline$w_{l} / \mathrm{mm}:$ & 11.574, & 9.962 & 8.137, & 6.538 & 5.433 & 4.850 \\
\hline$l_{t} / \mathrm{mm}:$ & 9.795 & 8.935, & 8.881 , & 9.689 & 9.442, & $0.0^{*}$ \\
\hline
\end{tabular}

Fig. 4: Transformer, $t=6.5 \mathrm{~mm}$

$* l_{t}=0$ indicates the output finned waveguide 
TABLE I (Continued)

Fig. 6: Transformer, $t=2.4 \mathrm{~mm}$

Input waveguide: WR-42, $a_{1}=10.688 \mathrm{~mm}, b_{1}=4.318 \mathrm{~mm}$

$b_{l} / \mathrm{mm}: \quad 4.299, \quad 4.261, \quad 4.223$,

$w_{\imath} / \mathrm{mm}: \quad 3.959, \quad 3.408, \quad 3.783$,

$l_{t} / \mathrm{mm}: \quad 3.667, \quad 3.345$,

3.313 ,

10.022

9.837

9.745

$* l_{t}=0$ indicates the output finned waveguide

Fig. 7: Transformer, $t=0.1 \mathrm{~mm}$

\begin{tabular}{|c|c|c|c|c|c|c|c|c|}
\hline \multicolumn{9}{|c|}{ Input waveguide: WR-15, $a_{1}=3.759 \mathrm{~mm}, b_{1}=1.880 \mathrm{~mm}$} \\
\hline $\begin{array}{l}a_{t} / \mathrm{mm}: \\
b_{t} / \mathrm{mm}: \\
w_{t} / \mathrm{mm}: \\
l_{t} / \mathrm{mm}:\end{array}$ & $\begin{array}{l}3.713 \\
1.856 \\
1.658 \\
1.559\end{array}$ & $\begin{array}{l}3.619 \\
1.809 \\
1.266 \\
1.304\end{array}$ & $\begin{array}{l}3.524, \\
1.762, \\
1.054, \\
1.347,\end{array}$ & $\begin{array}{l}3.430 \\
1.715 \\
0.791 \\
1.318\end{array}$ & $\begin{array}{l}3.336, \\
1.668, \\
0.603, \\
1.607\end{array}$ & $\begin{array}{l}3.241, \\
1.621, \\
0.503, \\
1.100,\end{array}$ & $\begin{array}{l}3.147 \\
1.574, \\
0.447 \\
1.642,\end{array}$ & $\begin{array}{l}3.100 \\
1.550 \\
0.400 \\
0.0^{*}\end{array}$ \\
\hline
\end{tabular}

Fig. 8: Transformer, $t=0.1 \mathrm{~mm}$

Input waveguide: WR-12, $a_{1}=3.099 \mathrm{~mm}, b_{1}=1.549 \mathrm{~mm}$

$l_{1}=1.299 \mathrm{~mm}$,

$w_{1}=1.283 \mathrm{~mm}$,

$w_{3}=0.831 \mathrm{~mm}$,

4.184,

4.146

4.127

$=1.468 \mathrm{~mm}$

$w_{5}=0.482 \mathrm{~mm}$,

$l_{2}=1.455 \mathrm{~mm}$

$l_{4}=1.291 \mathrm{~mm}$

$l_{6}=0.000 \mathrm{~mm} *$

$w_{2}=1.029 \mathrm{~mm}$

$w_{4}=0.653 \mathrm{~mm}$

$l_{5}=1.201 \mathrm{~mm}$,

finned waveguide

Fig. 9: Corrugated ridge waveguide filter, $t=1 \mathrm{~mm}$

Input waveguide: WR-75, R-120, $a_{1}=19.05 \mathrm{~mm}, b_{1}=9.525 \mathrm{~mm}$

$\begin{array}{lrrrrrrc}a_{\imath} / \mathrm{mm}: & 18.263 & 16.688 & 15.900 & 15.900 & 15.900 & 15.900 & 15.900 \\ & 15.900 & 15.900 & 15.900 & 15.900 & 15.900 & 15.900 & 15.900^{*} \\ b_{1} / \mathrm{mm}: & 9.131 & 8.344 & 7.950 & 7.950 & 7.950 & 7.950 & 7.950 \\ & 1.950 & 7.950 & 7.950 & 7.950 & 7.950 & 7.950 & 7.950^{*} \\ w_{l} / \mathrm{mm}: & 7.351 & 5.042 & 3.626 & 2.752 & 7.950 & 2.254 & 7.950 \\ & 1.758 & 7.950 & 1.537 & 7.950 & 1.430 & 7.950 & 1.429^{*} \\ l_{l} / \mathrm{mm}: & 8.403 & 4.946 & 7.954 & 4.013 & 1.402 & 6.127 & 1.652 \\ & 6.346 & 1.529 & 6.529 & 1.403 & 6.629 & 1.378 & 6.726^{*}\end{array}$

*Structure symmetric to the middle section

Fig. 10(a): Corrugated ridge waveguide filter, $t=0.3 \mathrm{~mm}$

\begin{tabular}{llcccccc}
\hline \multicolumn{2}{l}{ Input waveguide: $\mathrm{WR}-22, \mathrm{R}-400, a_{1}=5.689 \mathrm{~mm}, b_{1}=2.845 \mathrm{~mm}$} \\
$a_{\imath} / \mathrm{mm}:$ & 5.470 & 4.998 & 4.775 & 4.775 & 4.775 & 4.775 & 4.775 \\
& 4.775 & 4.775 & 4.775 & 4.775 & 4.775 & 4.775 & $4.775^{*}$ \\
$b_{l} / \mathrm{mm}:$ & 2.735 & 2.499 & 2.387 & 2.387 & 2.387 & 2.387 & 2.387 \\
& 2.387 & 2.387 & 2.387 & 2.387 & 2.387 & 2.387 & $2.387^{*}$ \\
$w_{l} / \mathrm{mm}:$ & 2.203 & 1.514 & 1.084 & 0.826 & 2.387 & 0.677 & 2.387 \\
& 0.528 & 2.387 & 0.461 & 2.387 & 0.429 & 2.387 & $0.429 *$ \\
$l_{l} / \mathrm{mm}:$ & 2.514 & 1.480 & 2.389 & 1.205 & 0.421 & 1.840 & 0.496 \\
& 1.906 & 0.459 & 1.961 & 0.421 & 1.991 & 0.414 & $2.020^{*}$
\end{tabular}

*Structure symmetric to the middle section

Fig. 10(b): Corrugated ridge waveguide filter, $t=0.3 \mathrm{~mm}$ Input waveguide: WR-22, R-400, $a_{1}=5.689 \mathrm{~mm}, b_{1}=2.845 \mathrm{~mm}$

\begin{tabular}{llllllll}
\hline$a_{i} / \mathrm{mm}:$ & 5.470 & 4.998 & 4.775 & 4.775 & 4.775 & 4.775 & 4.775 \\
& 4.775 & 4.775 & 4.775 & 4.775 & 4.775 & 4.775 & $4.775 *$ \\
$b_{i} / \mathrm{mm}:$ & 2.735 & 2.499 & 2.387 & 2.387 & 2.387 & 2.387 & 2.499 \\
& 2.387 & 2.617 & 2.387 & 2.735 & 2.387 & 2.844 & $2.387 *$ \\
$w_{i} / \mathrm{mm}:$ & 2.193 & 1.528 & 1.968 & 0.747 & 2.354 & 0.663 & 2.479 \\
& 0.489 & 2.606 & 0.451 & 2.698 & 0.486 & 2.841 & $0.425^{*}$ \\
$l_{i} / \mathrm{mm}:$ & 2.506 & 1.448 & 2.400 & 1.195 & 0.332 & 1.796 & 0.388 \\
& 1.860 & 0.406 & 1.915 & 0.352 & 1.999 & 0.360 & $2.136^{*}$
\end{tabular}

*Structure symmetric to the middle section 
TABLE I (Continued)

Fig. 11: Evanescent-mode filter, $t=0.2 \mathrm{~mm}$ Input waveguide: WR-19, $a_{1}=4.775 \mathrm{~mm}, b_{1}=2.387 \mathrm{~mm}$

\begin{tabular}{|c|c|c|c|c|c|c|}
\hline$a_{\imath} / \mathrm{mm}:$ & 2.228 & 2.228 & 2.228 & 2.228 & 2.228 & $2.228^{*}$ \\
\hline$b, / \mathrm{mm}:$ & 0.902 & 0.902 & 0.902 & 0.902 & 0.902 & $0.902 *$ \\
\hline w/mm: & 0.231 & 0.902 & 0.224 & 0.901 & 0.205 & $0.899 *$ \\
\hline$l / \mathrm{mm}:$ & 0.694 & 2.517 & 1.061 & 3.17 & 0.884 & $3.396 *$ \\
\hline
\end{tabular}

*Structure symmetric to the middle section

\section{APPENDIX}

Assuming electric and magnetic walls in Fig. 2(a) at $b / 2$ and $a / 2$, respectively, and indicating the slot region by $I I a$ and the adjacent region to the right by $I I b$, the crosssection functions $T_{H q}^{I I}, T_{E p}^{I I}$ for the finned waveguide read

$$
\begin{aligned}
T_{H q}^{I I}= & \sum_{n=0}^{N} Q_{h n}^{I I a} \cos \left\{\frac{2 n \pi}{w}(y-c)\right\} / \sqrt{1+\delta_{\text {on }}} \\
& +Q_{h n}^{I l b} \cos \left(\frac{2 n \pi}{b} y\right) / \sqrt{1+\delta_{\text {on }}} \\
T_{E p}^{I I}= & \sum_{n=1}^{N} P_{e n}^{I I a} \sin \left\{\frac{2 n \pi}{w}(y-c)\right\}+P_{e n}^{I I b} \sin \left(\frac{2 n \pi}{b} y\right)
\end{aligned}
$$

where $\delta_{\text {on }}$ is the Kronecker delta and

$$
\begin{aligned}
& Q_{h n}^{i}=A_{n}^{i} \exp \left(+j k_{x n}^{i} x\right)+B_{n}^{i} \exp \left(-j k_{x n}^{i} x\right) \\
& P_{e n}^{i}=\frac{1}{j k_{x n}^{i}}\left[C_{n}^{i} \exp \left(+j k_{x n}^{i} x\right)-D_{n}^{i} \exp \left(-j k_{x n}^{i} x\right)\right]
\end{aligned}
$$

$i=I I a, I I b . k_{x n}^{l}$ are the separation constants given by

$$
\left[\begin{array}{c}
\left(k_{x n}^{I I a}\right)^{2} \\
\left(k_{x n}^{I l b}\right)^{2}
\end{array}\right]=\omega^{2} \mu_{0} \epsilon_{0}-\left[\begin{array}{c}
\left(\frac{2 n \pi}{w}\right)^{2} \\
\left(\frac{2 n \pi}{b}\right)^{2}
\end{array}\right]-\left(k_{z H q, E_{p}}^{I I}\right)^{2} .
$$

By matching the partial waves (A3), (A4) and their derivatives

$$
P_{h n}^{i}=d Q_{h n}^{i} / d x, \quad Q_{e n}^{i}=d P_{e n}^{i} / d x
$$

at the interface at $e=(a-t) / 2$ (c.f. Fig. 2(a)) and applying the transverse resonance transformation, e.g. [21]-[22], two sets of matrix equations are obtained.

$$
\begin{aligned}
\underline{P}_{h(x=(a / 2))=}^{I l a} & {\left[\frac{b}{4} \operatorname{Diag}\left\{\cos \left(k_{x n}^{I I a} \frac{t}{2}\right)\right\} \underline{J}_{c}^{-1}\right.} \\
& \cdot \operatorname{Diag}\left\{-k_{x n}^{I l b} \sin \left(k_{x n}^{I b} e\right)\right\} \\
& +\frac{4}{w} \operatorname{Diag}\left\{-k_{x n}^{I I a} \sin \left(k_{x n}^{I I a} \frac{t}{2}\right)\right\} \underline{J}_{c} \\
& \left.\cdot \operatorname{Diag}\left\{\cos \left(k_{x n}^{I l b} e\right)\right\}\right] \cdot Q_{h(x=0)}^{I l b}
\end{aligned}
$$

$$
\begin{aligned}
& {\left[\frac{b}{4} \operatorname{Diag}\left\{\frac{1}{k_{x n}^{I l a}} \sin \left(k_{x n}^{I l a} \frac{t}{2}\right)\right\} \underline{J}_{c}^{-1} \operatorname{Diag}\left\{-k_{x n}^{I l b} \sin \left(k_{x n}^{I I b} e\right)\right\}\right.} \\
& \left.+\frac{4}{w} \operatorname{Diag}\left\{\cos \left(k_{x n}^{I I a} \frac{t}{2}\right)\right\} \underline{J}_{c} \operatorname{Diag}\left\{\cos \left(k_{x n}^{I I b} e\right)\right\}\right] \\
& \text { - } \underline{Q}_{h(x=0)}^{I l b}=0 \\
& \underline{P}_{e(x=(a / 2))}^{I l a}=\left[\frac{b}{4} \operatorname{Diag}\left\{\cos \left(k_{x n}^{I I a} \frac{t}{2}\right)\right\} \underline{J}_{s}^{-1}\right. \\
& \text { - Diag }\left\{\frac{1}{k_{x n}^{I l b}} \sin \left(k_{x n}^{I l b} e\right)\right\} \\
& +\frac{4}{w} \operatorname{Diag}\left\{\frac{1}{k_{x n}^{I l}} \sin \left(k_{x n}^{I l a} \frac{t}{2}\right)\right\} \underline{J}_{s} \\
& \text { - Diag } \left.\left\{\cos \left(k_{x n}^{I l b} e\right)\right\}\right] \cdot \underline{Q}_{e(x=0)}^{I l b} \\
& {\left[\frac{b}{4} \operatorname{Diag}\left\{-k_{x n}^{I l a} \sin \left(k_{x n}^{I l a} \frac{t}{2}\right)\right\} \underline{J}_{s}^{-1} \operatorname{Diag}\left\{\frac{1}{k_{x n}^{I l b}} \sin \left(k_{x n}^{I l b} e\right)\right\}\right.} \\
& \left.+\frac{4}{w} \operatorname{Diag}\left\{\cos \left(k_{x n}^{I I a} \frac{t}{2}\right)\right\} \underline{J}_{s} \operatorname{Diag}\left\{\cos \left(k_{x n}^{I l b} e\right)\right\}\right] \\
& \text { - } Q_{e(x=0)}^{I I b}=0
\end{aligned}
$$

with matrices $\underline{J}_{c}, \underline{J}_{s}$ given by

$$
\begin{aligned}
& \left(\underline{J}_{c}\right)_{m n}=\int_{c}^{b / 2} \frac{\cos \left(\frac{2 m \pi}{b} y\right) \cos \left\{\frac{2 n \pi}{w}(y-c)\right\}}{\sqrt{1+\delta \text { om }}} \frac{\sqrt{1+\delta \text { on }}}{\left.\sqrt{1+A^{\prime}} 11\right)} \\
& \left(\underline{J}_{s}\right)_{m n}=\int_{c}^{b / 2} \sin \left(\frac{2 m \pi}{b} y\right) \sin \left\{\frac{2 n \pi}{w}(y-c)\right\} d y
\end{aligned}
$$

(A8) and (A10) are the characteristic equations for the finned waveguide TE and TM modes, respectively, which need to be solved for cutoff frequencies $\left(k_{z H q, E p}=0\right)$. Together with (A3), (A4), (A6), the amplitude vectors in (A7)-(A10) specify functions $Q_{h n}^{i}$ and $P_{e n}^{i}$ in (A1), (A2), thus determining the cross-section functions for the finned waveguide. 


\section{REFERENCES}

[1] S. Hopfer, "The design of ridged waveguide," IRE Trans. Microwave Theory Tech., vol. MTT-3, pp. 20-29, Oct. 1955.

[2] E. S. Hensperger, "Broad-band stepped transformers from rectangular to double-ridged waveguide," IRE Trans. Microwave Theory Tech., vol. MTT-6, pp. 311-314, July 1955.

[3] J. P. Montgomery, "On the complete eigenvalue solution of ridged waveguide,"' IEEE Trans. Microwave Theory Tech., vol. MTT-19, pp. 547-555, June 1971 .

[4] W. J. R. Hoefer and M. N. Burtin, "Closed-form expressions for the parameters of finned and ridged waveguides," IEEE Trans. Microwave Theory Tech., vol. MTT-30, pp. 2190-2194, Dec. 1982.

[5] Y. Utsumi, "Variational analysis of ridged waveguide modes," IEEE Trans. Microwave Theory Tech., vol. MTT-33, pp. 111-120, Feb. 1985.

[6] Y. Konishi and H. Matsumura, "Short and effect of ridge guide with planar circuit mounted in a waveguide,"' IEEE Trans. Microwave Theory Tech., vol. MTT-27, pp. 168-170, Feb. 1979.

[7] D. Mirshekar-Syahkal and J. B. Davies, "Accurate analysis of tapered planar transmission lines for microwave integrated circuits,", IEEE Trans. Microwave Theory Tech., vol. MTT-29, pp. 123-128, Feb. 1981.

[8] L.-P. Schmidt and H. Meinel, "Broadband millimeter-wave PINdiode attenuator with double-ridged waveguide flanges,"' Electron. Lett., vol. 18, no. 19, pp. 839-840, Sept. 1982.

[9] P. J. Meier, "Integrated finline: The second decade," Microwave J., vol. 28 , no. 11, pp. 31-54, Nov. 1985; also no. 12, pp. 30-48, Dec. 1985.

[10] A. M. K. Saad, "Novel lowpass harmonic filters for satellite application," in IEEE MTT-S Int. Microwave Symp. Dig., 1984, pp. 292294.

[11] Q. Zhang and T. Itoh, "Computer-aided design of evanescent-mode waveguide filter with non-touching E-plane fins," IEEE Trans. Microwave Theory Tech., vol. 36, pp. 404-412, Feb. 1988.

[12] J. Bornemann and F. Arndt, "Modal-S-matrix design of optimum stepped ridged and finned waveguide transformers,"' IEEE Trans. Microwave'Theory Tech., vol. MTT-35, pp. 561-567, June 1987.

[13]. R. R. Mansour, R. S. K. Tong, and R. H. McPhie, "Simplified description of the field distribution in finlines and ridge waveguides and its application to the analysis of E-plane discontinuities," IEEE Trans. Microwave Theory Tech., vol. 36, pp. 1825-1832, Dec. 1988.

[14] H. E. Chappel, "Waveguide lowpass filter using evanescent mode inductors," Microwave J., vol. 21, pp. 71-72, Dec. 1978.

[15] G. Graven and C. K. Mok, "The design of evanescent mode waveguide band-pass filters for a prescribed insertion loss characteristic,", IEEE Trans. Microwave Theory Tech., vol. MTT-19, pp. 295-308, 1971 .

[16] R. V. Snyder, "New applications of evanescent mode waveguide to filter design," IEEE Trans. Microwave Theory Tech., vol. MTT-25, pp. 1013-1021, Dec. 1977.

[17] R. V. Snyder, "Broadband waveguide or coaxial filters with wide stopbands, using a stepped-wall evanescent mode approach," Microwave J., vol. 26, pp. 53-58, Dec. 1983.

[18] J. Bornemann and F. Arndt, "Rigorous design of evanescent-mode E-plane finned waveguide bandpass filters," in 1989 IEEE MTT-S Int. Microwave Symp. Dig., pp. 603-606, June 1989.

[19] T. N. Anderson, "Rectangular and ridge waveguide," IRE Trans. Microwave Theory Tech., vol. MTT-4, pp. 201-209, Oct. 1956.

[20] S. B. Cohn, "Optimum design of stepped transmission-line transformers,"'IRE Trans. Microwave Theory Tech., vol. MTT-3, pp. 1621, Apr. 1955.

[21] F. Arndt and G. U. Paul, "The reflection definition of the characteristic impedance of microstrips," IEEE Trans. Microwave Theory Tech., vol. MTT-27, pp. 724-731, Aug. 1979.

[22] R. Vahldieck and J. Bornemann, "A modified mode-matching technique and its application to a class of quasi-planar transmission-lines," IEEE Trans. Microwave Theory Tech., vol. MTT-33, pp. 916-926, Oct. 1985 .

[23] R. Sorrentino and T. Itoh, "Transverse resonance analysis of fin-line discontinuities,"' IEEE Trans. Microwave Theory Tech., vol. MTT32, pp. 1633-1638, Dec. 1984.

[24] A. Wexler, "Solution of waveguide discontinuities by modal analysis," IEEE Trans. Microwave Theory Tech., vol. MTT-15, pp. 508517 , Sept. 1967.
[25] E. Kühn, "A mode-matching method for solving field problems in waveguide and resonator circuits,", Arch. El. Übertragungstech., vol. 27, pp. 511-518, Dec. 1973.

[26] R. Vahldieck, J. Bornemann, F. Arndt, and D. Grauerholz, "Optimized waveguide E-plane metal insert filters for millimeter-wave applications," IEEE Trans. Microwave Theory Tech., vol, MTT-31, pp. 65-69, Jan. 1983.

[27] H. Patzelt and F. Arndt, "Double-plane steps in rectangular waveguides and their application for transformers, irises, and filters," IEEE Trans. Microwave Theory Tech., vol. MTT-30, pp. 771-776, May 1982.

[28] J.-W. Tao and H. Baudrand, "Multimodal variational analysis of uniaxial waveguide discontinuities," IEEE Trans. Microwave Tech., vol. 39, pp. 506-516, Mar. 1991.

[29] J. Bornemann, "Comparison between different formulations of the transverse resonance field-matching technique for the three-dimensional analysis of metal-finned waveguide resonators," Int. J. Numerical Modeling, vol: 4, pp. 63-73, Mar, 1991.

[30] J. Bornemann and F. Arndt, "Transverse resonance, standing wave, and resonator formulations of the ridge waveguide eigenvalue problem and its application to the design of E-plane finned waveguide filters," IEEE Trans. Microwave Theory Tech., vol. 38, pp. 11041113 , Aug. 1990.

[31] F. Arndt, T. Duschak, U. Papziner, and P. Rolappe, "Asymmetric iris coupled cavity filters with stopband poles," in IEEE MTT-S Int. Microwave Symp. Dig., pp. 215-218, 1990.

[32] F. Arndt, "Rigorous field theoretic computer-aided design of microwave and millimeter wave diplexers," in 1990 MIOP. Microwaves and Optronics Int. Conf. Proc., Stuttgart, Apr. 1990, pp. 44-53,

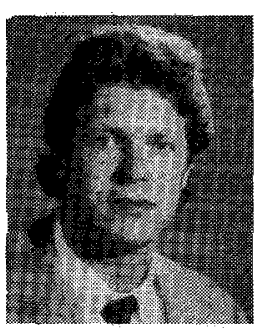

Jens Bornemann (M'87-SM'90) was born in Hamburg, West Germany, on May 26, 1952. He received the Dipl.-Ing. and the Dr.-Ing. degrees, both in electrical engineering, from the University. of Bremen, West Germany, in 1980 and 1984, respectively.

From 1980 to 1983 , he was a Research and Teaching Assistant in the Microwave Department at the University of Bremen, working on quasiplanar waveguide configurations and computeraided E-plane filter design. After a two year period as a consulting engineer, he joined the University of Bremen again, in 1985, where he was employed at the level of Assistant Professor. Since April 1988, he has been an Associate Professor at the University of Victoria, Victoria, BC, Canada. His current research activities include microwave system design and problems of electromagnetic field theory in integrated circuits and radiating structures.

Dr. Bornemann was one of the recipients of the A.F. Bulgin Premium of the Institution of Electronic and Radio Engineers in 1983. He serves on the editorial board of the IEEE TRANSACTIONS ON MICROWAVE THEORY AND TECHNIQues, and has authored and coauthored more than 50 technical papers.

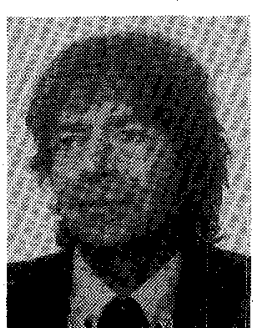

Fritz Arndt (SM'83) received the Dipl.-Ing., Dr.Ing., and Habilitation degrees from the Technical University of Darmstadt, Germany, in 1963, 1968 , and 1972 , respectively.

From 1963 to 1973 , he worked on directional couplers and microstrip techniques at the Technical University of Darmstadt. Since 1972, he has been a Professor and Head of the Microwave Department of the University of Bremen, Germany. His research activities are in the area of the solution of field problems of waveguide, finline, optical waveguide structures, of anténna design, and of scattering structures. Dr. Andt is member of the VDE and NTG (Germany). He received the NTG award in 1970, the A. F. Bulgin Award (together with three coauthors) from the Institution of Radio and Electronic Engineers in 1983, and the best paper award of the antenna conference JINA 1986 (France). 